\title{
Erratum to: Enrollment and Attendance in a Parent Training Prevention Program for Conduct Problems
}

\author{
Courtney N. Baker • David H. Arnold • Susan Meagher
}

Published online: 29 January 2011

(C) Society for Prevention Research 2011

\section{Erratum to: Prev Sci}

DOI 10.1007/s11121-010-0187-0

In the introduction of our manuscript ("Enrollment and Attendance in a Parent Training Prevention Program for Conduct Problems") we wrote that: "the constructs of enrollment and attendance are often blended into one continuous measure, which washes out important distinctions between the constructs (e.g., Garvey et al. 2006; Gross et al. 2001)."

The cited studies are examples of research that demonstrated the importance of separating these constructs by doing so; these were groundbreaking studies that were among the first to disentangle the two constructs. However, our wording was ambiguous, and could be interpreted as meaning the opposite. We feel badly that we potentially implied the reverse of what this important research accomplished.

The online version of the original article can be found at http://dx.doi. org/10.1007/s11121-010-0187-0.

C. N. Baker $\cdot$ D. H. Arnold $(\varangle) \cdot$ S. Meagher

University of Massachusetts Amherst,

Amherst, MA, USA

e-mail: darnold@psych.umass.edu 